"Managing investment and liquidity risks for derivatives within a market impact perspective"

\begin{tabular}{|c|c|}
\hline AUTHORS & Aymeric Kalife \\
\hline ARTICLE INFO & $\begin{array}{l}\text { Aymeric Kalife (2017). Managing investment and liquidity risks for derivatives } \\
\text { within a market impact perspective. Insurance Markets and Companies, 8(1), 59- } \\
\text { 73. doi:10.21511/ins.08(1).2017.06 }\end{array}$ \\
\hline DOI & http://dx.doi.org/10.21511/ins.08(1).2017.06 \\
\hline RELEASED ON & Wednesday, 27 December 2017 \\
\hline RECEIVED ON & Thursday, 02 November 2017 \\
\hline \multirow[t]{2}{*}{ ACCEPTED ON } & Wednesday, 06 December 2017 \\
\hline & $(\mathrm{cc})$ EY-NC \\
\hline LICENSE & $\begin{array}{l}\text { This work is licensed under a Creative Commons Attribution-NonCommercial } 4.0 \\
\text { International License }\end{array}$ \\
\hline JOURNAL & "Insurance Markets and Companies" \\
\hline ISSN ONLINE & $2522-9591$ \\
\hline PUBLISHER & LLC "Consulting Publishing Company "Business Perspectives" \\
\hline FOUNDER & LLC "Consulting Publishing Company "Business Perspectives" \\
\hline & 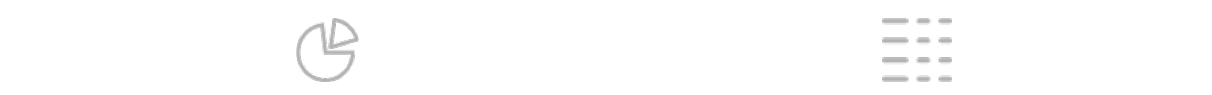 \\
\hline NUMBER OF REFERENCES & NUMBER OF FIGURES \\
\hline 22 & 12 \\
\hline
\end{tabular}

(C) The author(s) 2022. This publication is an open access article. 
Aymeric Kalife (France)

\title{
Managing investment and liquidity risks for derivatives within a market impact perspective
}

\begin{abstract}
The recent period has experienced many instances when market volatility suddenly increased even when there were no well-known fundamental catalysts, as illustrated by the short-lived but sharp transitions from low volatility to high volatility, as many in the last six years as we have had in the prior two decades - increasing evidence that we are in a new volatility-of-volatility regime. Fundamentally, market impact is an illustration of market inefficiency: theories of efficient markets typically expect that investors buy and sell assets based on assessments of their intrinsic value, in contrast with large derivative players who often act based on market price movements which may not be linked to fundamentals. Market impact risk refers to the degree to which large size transactions can be carried out in a timely fashion with a minimal impact on prices. As a result, managing investment and liquidity risks for large players requires introducing an explicit market impact function, and applying to derivatives significantly depends on whether there is or not significant delta hedging activity: in case of no significant delta hedging activity, the risk appetite has significant influence on the optimal execution strategy, while in case of significant delta hedging activity the optimal trading involves feedback hedging effects translating into a modified Black - Scholes hedging strategy.
\end{abstract}

Keywords: market impact, liquidity, transaction size, hedging, optimal trading.

JEL Classification: G11, G13, G14.

Received on: $2^{\text {nd }}$ of November, 2017.

Accepted on: $6^{\text {th }}$ of December, 2017.

\section{Introduction}

The short-lived but severe volatility spikes experienced over the past couple of years are not done, especially with low liquidity and low conviction. Sharp transitions from low volatility

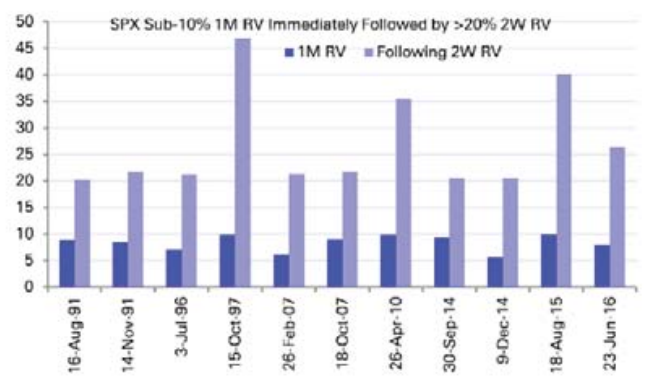

Fig. 1. Almost as many transitions from lows to highs over the past 7 years as in the prior 20 years

Source: Deutsche Bank, Bloomberg Finance LP.

Market liquidity risk refers to the degree to which large size transactions can be carried out in a timely mannes with a minimal impact on prices. Fundamentally, market impact is an illustration of market inefficiency: actually, theories of efficient markets typically expect that investors

(C) Aymeric Kalife, 2017.

Aymeric Kalife, Ph.D., Adjunct Professor of Finance, University of Paris Dauphine, France.

This is an Open Access article, distributed under the terms of the Creative Commons Attribution-NonCommercial 4.0 International license, which permits re-use, distribution, and reproduction, provided the materials aren't used for commercial purposes and the original work is properly cited to high volatility are becoming increasingly common, as many in the last six years as we have had in the prior two decades - increasing evidence that we are in a new volatility-of-volatility regime.

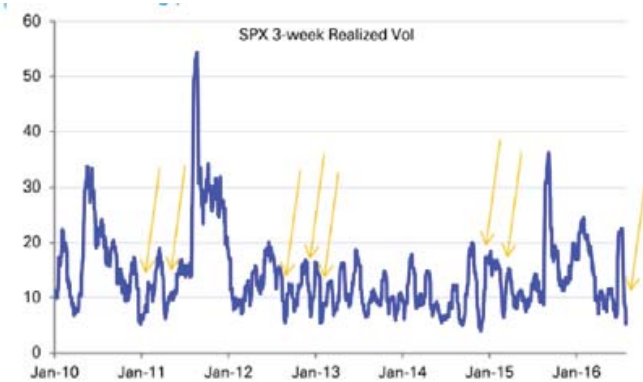

Fig. 2. Sudden drops in SPX realized volatility from high levels to very low levels have become increasingly common

Source: Deutsche Bank, Bloomberg Finance LP.

are buying and selling assets based on assessments of their value; in contrast, large derivatives players buy and sell based on observations of market price movements, thus have a utility function quite different from that of value investor.

Although fears about growth or sovereign debts sustainability are valid explanations for significant declines in equities as experienced in May 2010 (caused by the Greece sovereign debt crisis threatening the credibility and sustainability of the European Union Monetary Union), August 2011 (triggered by the US government debt ratings downgrade by Standard \& Poor's agency), August 2015 (due to strong concerns about China economic 
growth) or February 2016 (due to lower than expected China economic growth), they do not fully explain neither the extreme magnitude of the shocks nor the repeated occurrence at the close in European and US markets. The recent period has experienced many instances when market volatility suddenly increased even when there were no well-known fundamental catalysts.

Actually, derivatives activity by large players might help exacerbate the acuity of such volatility spikes from the illiquidity premium in option markets since the nineties (the late 1990's bull market with overwriting supplied volatility from pressure for income generation, or the period 1997-2000 with strong demand for bond + call participation rates). An empirical study conducted by Christoffersen et al. (2014) presented strong evidence of this, stemming from a structural imbalance between supply and demand in derivatives as illustrated by:

- USD 5 trillion outstanding equity derivatives in the US: USD 3 trillion listed, i.e., 20-fold since 2000, above the cash equity volume during 2005-2007; USD 2 trillion in the OTC market in 2005 before $40 \%$ drop since 2008 .

- Structural supply-demand imbalance in shortdated volatility: $70 \%$ more put options outstanding than call outstanding, with directional users buying puts for portfolio protection; growth of the variance swap market requiring short positions in deep-outof-the money puts.

- Structural supply-demand imbalance in longdated volatility:

1. from US variable annuities and Asian structured products hedging, translating into steepening of the term structure;

2. from hedging US mortgages convexity (homeowners own the option to prepay, valuable when interest rates fall, leaving the market net short volatility): USD 900 billion options hedge in 2005 (vs. USD 200 billion in 1993.

Given such suppy-demand imbalance in derivatives, the cost of placing one large order to close a position will be far greater than the sum of infinitely small orders differed in time. For this reason, an explicit modeling is required through a market impact function, the influence of which the agent will try to minimize. The optimal execution turns out to be the sequence of small trades over the course of several days that optimizes a target, e.g., minimizes the mean cost of trading over a fixed period.
Such imbalance in the derivatives markets is also at the source of hedging inefficiencies, as illustrated by the impact of short gamma positions synthetic hedging, where market makers tend to sell more as the market drops or acquire additional long exposure as the market rallies, which may significantly contribute to market liquidity disruptions, independently of fundamentals and related to the growth of the options market, as illustrated by:

- The '02 sell-off inducing large-scale hedging amid extraordinary volatility and a spike in option prices.

- April 2005 decline in equity markets which was the largest two-day fall.

- The huge downward moves in October 2008 triggered the $70 \%$ barrier of knock-in put embedded within autocallables sold in Asia, where the delta hedging effects have exacerbated the moves on the downside as the barriers were triggered.

In this paper, we consider the optimal execution price and strategies of options when market impact is a driver of the option price, which depends on whether the delta hedging of the options is significant or not:

- In case of no or insignificant delta hedging (like for a life insurance company aiming to minimize the cost of buying a large quantity of put options to hedge liabilities), the optimal execution turns out to be strongly dependent on the risk appetite: in the mean cost minimization objective, the solution leads to a closed formula where the trading speed is of the inverse of a $3 / 2$ power law (as the maturity approaches the agent must acquire faster as time passes). In contrast, in the mean-variance case objective (where the dispersion of revenues is taken into account) the agent tends to liquidate her position at the beginning in order to reduce the $\mathrm{P} \& \mathrm{~L}$ variance.

- In case of significant delta hedging, the optimal execution strategy is determined by a no arbitrage framework that incorporates the specific impact of the large trader's hedging activity (so called "hedging feedback effects"), which translates into a fully nonlinear modified Black-Scholes delta hedging strategy.

In section 1, the most observed types of market impact on the investment and liquidity risks within derivatives strategies will be illustrated and analyzed from a qualitative perspective. Section 2 will be dedicated to the optimal strategies in derivatives based on appropriate modeling of the market impact, depending on 
whether the is significant associated delta hedging activity or not.

\section{Empirical market impact on the investment and liquidity risks within derivatives strategies}

1.1. Using derivatives enables to hedge financial risks embedded within liabilities. Insurance companies, corporates or asset managers utilize derivatives in a variety of ways to manage and mitigate risks that are inherent in their investment or liability portfolios which can be characterized by three main features: medium long-term duration, large volumes and significant market risk exposure.

Given the persistent low interest rate environment across the curve since the 2008 financial crisis, these large players need hedge their liabilities, as illustrated by the significant recent increase from USD 786 billion as of fiscal year 2010 to USD 1,885 billion as of FY 2014 by insurance companies. As the guarantees embedded within those liabilities hold a convex risk profile with respect to the underlying stock, they need to buy some convex equity hedge assets such as options in order to match the liability risk profile to improve hedge effectiveness: ninety percent of the put options were purchased, implying the growing cost of hedging.

As equity derivatives are highly sensitive to supply/demand balance, buying large hedge

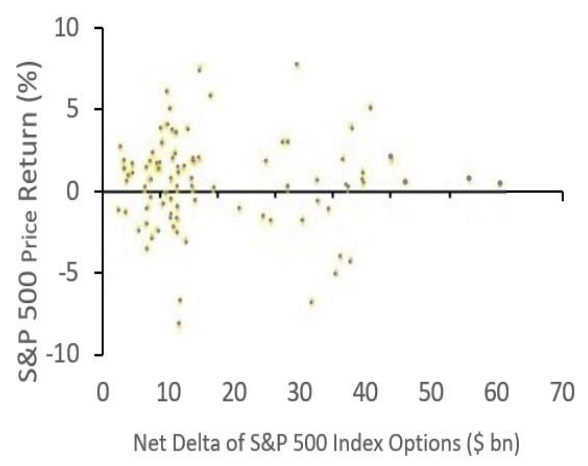

Fig. 3. Expiration week return vs. net-delta of front-month S\&P options on previous Friday

Source: Deutsche Bank.

1.2.2. Hedging feedback effects on long-dated options: focus on autocallables. Structured products are often upside (capped) participation with capital guaranteed (floor), seller is long ATM vol and short OTM vol at both wings.

As we approach maturity gamma becomes more localised around the strike, which tends to have greater effects on actual volatility in the spot market. It turns out that barriers can cause gamma to portfolios requires taking into account the transaction size explicitly, which is not explicitly considered by traditional models. Basel III and Solvency II are expected to further strengthen this illiquidity as they aim to match capital requirements with the economic risks embedded within the liabilities, thus requiring large quantities of options where prices will increase as a result of supply and demand imbalance.

1.2. Such large derivatives imbalances are likely to imply net short positions in options by market makers, thus synthetic replication with significant delta-hedging activity likely to exacerbate market moves through "hedging feedback effects".

1.2.1. Hedging feedback effects on short-dated vanilla options. Investors typically (i.e., 65-80 \%) buy index put as protection for downside protection, thus market makers short put options which they hedge by selling futures to be market neutral. If the market suddenly drops, they would need to sell further to adjust which amplifies the down market move. Options dealers (delta-hedgers) are net-short options against outright investors (non-deltahedgers), and are large enough to move the equity market, which might further exacerbate market moves thus volatility. Their total risk exposure amounts to $40 \%$ of the total gamma, i.e. USD 7 billion sold near market close for $1 \%$ move per day.

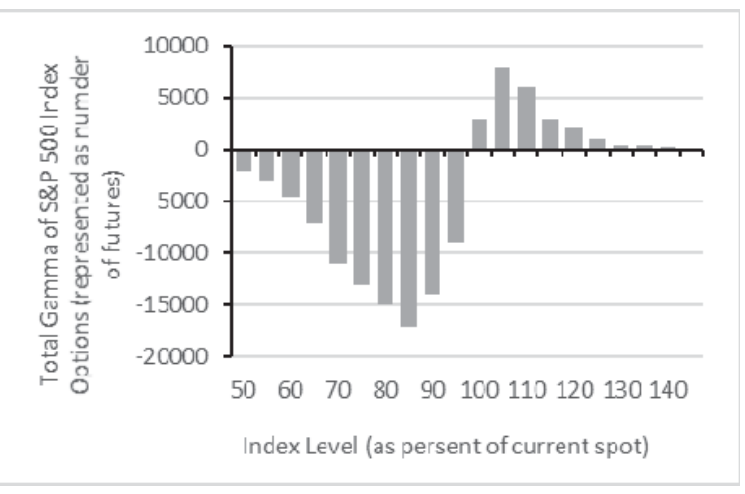

Fig. 4. Gamma as a function of spot S\&P 500 index options

reverse across very small movements as spot rallies towards the KO barrier, and create much larger gamma positions than vanilla strikes, particularly near to expiry. In this case, the trader will be selling large amounts of spot as it approaches the barrier, which will tend to prevent the spot market from actually hitting the barrier.

Despite this selling of spot and gamma, the barrier may at some point break. If this happens, the option 
disappears, and the trader is left only with his hedge, i.e., a naked position, which he has to cover by buying back spot and gamma, where delta-hedging will tend to exaggerate spot moves even more (spot higher $->$ needs to buy $->$ drives spot higher, spot lower $->$ needs to sell $->$ drives spot lower) which will cause the spot market to become more liable to choppy trading and can cause the market to gap higher.

Because of leverage in barrier options, the effect on vanilla market is disproportional to notional size: Closer to maturity, delta amounts grow to multiples of the size of the original option.

\section{Optimal derivatives strategies}

2.1. In case of no significant delta hedging activity, the optimal execution turns out to be strongly dependent on risk appetite. Here we consider that delta hedging is either nil or negligible in terms of market impact, which is consistent with practice on the main market indices as their exchanged volumes is far larger than for the corresponding options contracts, as illustrated below.

Table 1. S\&P 500 Historical Volume Data (2 Jan. 1951 - 31 Mar. 2012)

\begin{tabular}{|c|c|c|c|c|}
\hline & Total shares & Avg. shares & Correlation & $\mathrm{R}^{2}$ \\
\hline $1950 \mathrm{~s}$ & $5,777,550,000$ & $2,298,150$ & 0.66 & 0.44 \\
\hline $1960 \mathrm{~s}$ & $19,072,060,000$ & $7,656,387$ & 0.73 & 0.53 \\
\hline $1970 \mathrm{~s}$ & $57,655,100,000$ & $22,833,703$ & 0.48 & 0.23 \\
\hline $1980 \mathrm{~s}$ & $306,188,530,000$ & $121,118,881$ & 0.76 & 0.58 \\
\hline $1990 \mathrm{~s}$ & $1,195,610,210,000$ & $473,134,234$ & 0.93 & 0.86 \\
\hline $2000 \mathrm{~s}$ & $7,091,918,888,000$ & $2,819,848,464$ & $0.07)$ & 0.01 \\
\hline $2010 \mathrm{~s}$ & $1,274,419,730,000$ & $4,058,661,561$ & $0.32)$ & 0.1 \\
\hline Total & $9,950,642,068,000$ & & 0.72 & 0.52 \\
\hline
\end{tabular}

Source: Yahoo Finance. CFA Institute.

The average shares traded per day for the S\&P 500 has grown from 2.3 million to 4.1 billion, with as busiest trading day ever for the S\&P 500 the 10
October 2008, when a phenomenal 11,456,230,400 shares changed hands. In contrast options contracts exchanged volumes are significantly lower.

Table 2. Listed options - most actively traded and open interest - S\&P500 index options

\begin{tabular}{|c|c|c|c|c|}
\hline \multicolumn{5}{|c|}{ Listed options - most actively traded (average over past week) } \\
\hline & Expiry & Strike & Number of contracts & $\begin{array}{l}\text { Notional value of contracts } \\
\text { (MM, local currency) }\end{array}$ \\
\hline PUT & June 16, 2017 & $2,375.0$ & 13,007 & $3,088.1$ \\
\hline PUT & April 21, 2017 & $2,300.0$ & 12,793 & $3,033.1$ \\
\hline CALL & April 21, 2017 & $2,375.0$ & 12,322 & $2,931.2$ \\
\hline CALL & June 16, 2017 & $2,375.0$ & 12,071 & $2,865.7$ \\
\hline PUT & April 21, 2017 & $2,310.0$ & 10,108 & $2,404.9$ \\
\hline \multicolumn{5}{|c|}{ Listed options - largest open interest } \\
\hline & Expiry & Strike & Number of contracts & $\begin{array}{l}\text { Notional value of contracts } \\
\text { (MM, local currency) }\end{array}$ \\
\hline PUT & June 16, 2017 & $2,000.0$ & 81,710 & $19,153.0$ \\
\hline CALL & April 21, 2017 & $2,375.0$ & 76,091 & $17,835.9$ \\
\hline PUT & April 21, 2017 & $2,200.0$ & 75,310 & $17,652.8$ \\
\hline PUT & April 21, 2017 & $2,300.0$ & 75,147 & $17,614.6$ \\
\hline PUT & April 21, 2017 & $2,375.0$ & 65,530 & $15,360.0$ \\
\hline
\end{tabular}

Source: Deutsche Bank.

An agent who is willing to trade a large quantity of options will see the impact as an important dilemma, as the cost of placing one large order to close his position will be far greater than the sum of infinitely small orders differed in time. In practice they are usually broken up into smaller ones and executed over the course of several days (see for example, Chan \& Lakonishok, 1995 \& Keim \& Madhavan 1995): only 20\% of the market value of the trades splits in their set of data are completed within a day, and that over 53\% are spread over four trading days or more. For this reason, an explicit modeling is made through a market impact function (depends on the "temporary impact strength" that is proportional to the main empirically observed drivers such as the speed of option trading, i.e., the number of options per unit of time, the equity stock level and the option sensitivity to the equity stock), the influence of which the agent will try to minimize. The optimal execution turns out to be the sequence of trades that optimizes the target, e.g., minimizes the mean cost of trading over a fixed period, or the mean-variance 
criterion if the volatility of revenues is taken into account.

2.1.1. The market impact function and resulting option price in case of no significant delta hedging. The model is inspired from Leland's option replication with transaction costs that is incorporated into the option price as an additional variable within the volatility function:

$$
\tilde{\sigma}^{2}=\sigma^{2}+f\left(t, \dot{x}_{t}, x_{t}, \sigma\right) .
$$

where $\sigma$ is the asset volatility and $\mathrm{f}$ is the market impact function (depends on time, volatility, inventory and trading speed). The option effective price is then expressed through a Black-Scholes like PDE with such modified "enlarged" volatility in order to compensate for the market impact cost. Finally, regarding the best execution strategy, we set up a stochastic control framework and solve a Hamilton-Jacobi-Bellman equation using finite differences methods.

In terms of market impact function, we follow the approach by Almgren and Chriss (2000) and Almgren (2003) where the price impact is a combination of two components: a permanent component that reflects the information transmitted to the market by the buy/ sell imbalance; and a temporary component that reflects the price concession needed to attract counter-parties within a specified short time interval. The number of shares of the traded asset is described by an absolutely continuous trajectory $t \rightarrow x_{t}, \quad \dot{x}_{t}$, its derivative w.r.t time corresponds to the speed of trading of the security.

In the absence of market impact, the asset is modeled by a geometric Brownian motion (GBM): $S_{t}=S_{0} \cdot e^{-\frac{1}{2} \sigma^{2} t+\sigma W_{t}}$. When market impact is taken into account, the execution price is defined by $\tilde{S}_{t}=S_{t} \cdot\left(1+\eta \cdot \dot{x}_{t}+\gamma \cdot\left(x_{t}-x_{0}\right)\right)$, where $\mathrm{S}$ is the unaffected stock price process, and $r_{i}$ and $\gamma$ are constants. The term $\eta \cdot \dot{x}_{t}$ corresponds to the temporary or instantaneous impact of trading $\dot{x}_{t} d t$ shares at time $t$ and only affects this current order. The term $\gamma \cdot\left(x_{t}-x_{0}\right)$ is the permanent price impact which was accumulated by all transactions until time $\mathrm{t}$.

We adapt such formula to the "enlarged volatility" expression as follows:

$$
\tilde{\sigma}_{t}^{2}=\sigma^{2}+\left(\tilde{\eta} \cdot \dot{x}_{t}+\tilde{\gamma} \cdot\left(x_{t}-x_{0}\right)\right) \cdot \sqrt{\hat{T}-t \cdot \sigma},
$$

where $\tilde{\eta}=\eta \sqrt{\frac{8}{h \pi}}$ and $\tilde{\gamma}=\gamma \sqrt{\frac{8}{h \pi}}$. Finally, the option price under market impact follows the PDE:

$$
\left\{\begin{array}{l}
\partial_{u} \tilde{P}(u, S)+\frac{1}{2} \tilde{\sigma}_{t}^{2} \cdot S^{2} \partial_{s s} \cdot \tilde{P}(u, S)=0, \quad(u, S) \in[t, \hat{T}[\times] 0, \infty] \\
\tilde{P}(\hat{T}, s)=(K-s)+.
\end{array}\right.
$$

Using a simple Taylor approximation to the firstorder, we can rewrite the above expression as a sum of the Black-Scholes option price and an additional term corresponding to the option market impact:

$$
\tilde{P}\left(t, S_{t}\right) \approx P\left(t, S_{t}\right)+\left(\tilde{\sigma}_{t}^{2}-\sigma^{2}\right) \partial_{v} P\left(t, S_{t}\right) \approx P\left(t, S_{t}\right)+\frac{1}{2}\left\{\tilde{\eta} \cdot \dot{x}_{t}+\tilde{\gamma} \cdot\left(x_{t}-x_{0}\right)\right\} \cdot \sqrt{\hat{T}-t v\left(t, S_{t}\right)}
$$

where $v\left(t, S_{t}\right)=\partial_{\sigma} P$ is the Black-Scholes vega of the option:

$$
\begin{aligned}
& v\left(t, S_{t}\right)=\sqrt{\hat{T}-t S_{t} N^{\prime}}\left(d_{1}\right)=\sqrt{\hat{T}-t K N^{\prime}}\left(d_{2}\right) \\
& N^{\prime}(x)=\frac{1}{\sqrt{2 \pi}} e^{\frac{-x^{2}}{2}} \\
& d_{1}=\frac{\log \frac{S_{t}}{K}+\frac{1}{2} \sigma^{2}(\hat{T}-t)}{\sigma \sqrt{\hat{T}-t}}, \\
& d_{2}=\frac{\log \frac{S_{t}}{K}-\frac{1}{2} \sigma^{2}(\hat{T}-t)}{\sigma \sqrt{\hat{T}-t}}=d_{1}-\sigma \sqrt{\hat{T}-t}
\end{aligned}
$$

It follows that the market impact function is defined by 


$$
\int\left(t, S_{t}, \dot{x}_{t}, x_{t}\right):=\tilde{P}\left(t, S_{t}\right)-P\left(t, S_{t}\right)=\frac{1}{2}\left\{\tilde{\eta} \cdot \dot{x}_{t}+\tilde{\gamma} \cdot\left(x_{t}-x_{0}\right)\right\} \cdot \sqrt{\hat{T}-t v\left(t, S_{t}\right)}
$$

This allows to write the option execution price as the sum the option price in the absence of market impact plus a positive term reflecting the additional cost due to market impact. Using the Vega-Gamma relationship (i.e., $v=\sigma \tau S^{2} \Gamma$ we can rewrite the market impact term as a function of the option Gamma:

$$
\int\left(t, S_{t}, \dot{x}_{t}, x_{t}\right)=\frac{1}{2}\left\{\tilde{\eta} \cdot \dot{x}_{t}+\tilde{\gamma} \cdot\left(x_{t}-x_{0}\right)\right\} \sigma S_{t}^{2}(\hat{T}-t)^{\frac{3}{2}} \Gamma\left(t, S_{t}\right),
$$

where $\Gamma\left(t, S_{t}\right)=\frac{N^{\prime}\left(d_{1}\right)}{S_{t} \sigma \sqrt{\hat{T}-t}}=\frac{K N^{\prime}\left(d_{2}\right)}{S_{t}^{2} \sigma \sqrt{\hat{T}-t}}$,

$\tilde{\eta}$ controls the temprorary impact strength in USD $\times$ hour/N of options; $\tilde{\gamma}$ controls the permanent impact strength and in USD N shares; $x_{t}$ is the quality held at time $t$ and $x_{t}$ is the speed of trading in number of options per time unit; $\Gamma$ is the delta sensitivity w.r.t to the asset price

$\left(\Gamma\left(t, S_{t}\right)>0\right)$.

\section{Optimal Option Purchasing Strategy with Market Impact}

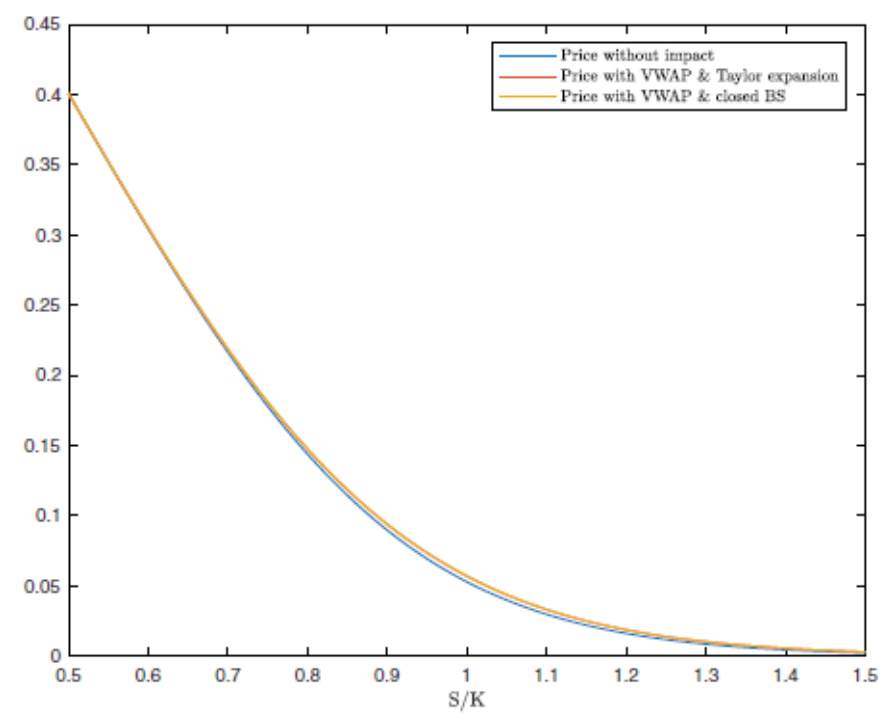

Fig. 5. Put price as a function of the moneyness

As illustrated above, the approximation by the Taylor expansion is almost equal to the closed formula, which will be very convenient to solve the optimal trade execution problem.

As a result the option price under market impact is found to verify a Black-Scholes like PDE with an "enlarged" volatility, where buying the option will typically lead to increasing its price, as expected: the higher the trading speed and quantity, the higher the volatility and thus the option price.

2.1.2. The optimal execution problem. As explained above, the optimal execution is a strategy that unfolds over the course of several days and which ought to adapt to changing market conditions. This is intuitively true for market options as well, even though very little literature deals with this issue. Taking this into an account, instead of executing his orders at once, the agent has to split them over the time interval $[0, T]$ by means of a dynamic order execution strategy.

Let us consider a buying trade execution strategy in which an initial long position of $X$ options with fixed strike $K$ and maturity $T$ is liquidated by a fixed time horizon $[0, T]$. We describe such a strategy by the asset position $x(t)$ held at time $t$. The initial position $\mathrm{x}(0)$ is negative for a buying strategy and the condition $x_{T+}=0$ assures that the initial position has been unwound by time $T$. The path $x=\left(x_{t}\right)_{t \in[0, T]}$ will be nondecreasing for a pure buying strategy. The enduser's purpose is to hedge the risk of a complex product (structured product, Variable Annuity, etc.) indexed on an underlying asset, by acquiring vanilla put options on that same underlying asset. 
Let $(\Omega, F, \mathrm{P})$ be the usual probability space on the filtration $\left(F_{t}\right)_{t \in R_{+}}$satisfying the usual assumptions. In the absence of market impact and under a null risk-free rate, the no-arbitrage price of a put option is defined by $P_{t}=E_{Q}\left[\left(K-S_{\hat{T}}\right)+\left|F_{t}\right|\right]$. under the risk-neutral probability measure $\mathrm{Q}$ in which the asset price is a martingale. At each time $t, \dot{x}_{t} d t$ options are bought at price $\tilde{P}_{t}$ which is the option impact price defined by the PDE or the price equation above. Thus, the cost arising from the strategy $\mathrm{x}$ is $C(x):=\int_{0}^{T} \tilde{P}_{t} \dot{x}_{t} d t$.

The agent's objective is then to minimize a certain objective function, which takes into account his risk aversion, and may involve both cost and risk terms, over the class of admissible trading strategies $\mathrm{x}$ with side conditions $x_{0}=X$ and $x_{T}=0$. This is known as the optimal trade execution problem. In this paper, we will consider two risk aversion cases:

- The mean cost $E[C(x)]$

- The mean-variance case $E[C(x)]+\lambda \operatorname{Var}[C(x)]$ (which includes the mean case with $\lambda=0$ )

We will develop the framework under the Black and Scholes case a temporary market impact only, with permanent impact excluded, i.e., $\tilde{\gamma}=0$. In that case, the effective price is given by:

$$
\tilde{P}_{t}=P_{t}+\frac{1}{2} \tilde{\eta} \dot{x}_{t} \sigma S_{t}^{2}(\hat{T}-t)^{\frac{3}{2}} \Gamma\left(t, S_{t}\right) .
$$

We can rewrite the cost function as follows:

$$
C(x)=\int_{0}^{T} P_{t} \dot{x}_{t} d t+\frac{1}{2} \tilde{\eta} \int_{0}^{T} \dot{x}_{t}^{2} \sigma S_{t}^{2}(\hat{T}-t)^{\frac{3}{2}} \Gamma\left(t, S_{t}\right) d t .
$$

Using a simple integration by part and Ito's formula, the cost arising from the strategy $x$ becomes

$$
C(x)=-X P_{0}-\int_{0}^{T} \sigma x_{t} S_{t} \Delta\left(t, S_{t}\right) d W_{t}+\frac{1}{2} \tilde{\eta} \sigma \int_{0}^{T} \dot{x}_{t}^{2} S_{t}^{2}(\hat{T}-t)^{\frac{3}{2}} \Gamma\left(t, S_{t}\right) d t,
$$

where $\Delta$ is the Black-Scholes delta of the option calculated on $\sigma$.

The mean cost of the strategy $x$ is then

$$
E[C(x)]=-X P_{0}+\frac{1}{2} \tilde{\eta} E\left[\int_{0}^{T} \dot{x}_{t}^{2} S_{t}^{2}(\hat{T}-t)^{\frac{3}{2}} \Gamma\left(t, S_{t}\right) d t\right] .
$$

Theorem 1. The optimal strategy $x^{*}$ resulting in minimizing the mean cost under the Black and Scholes framework is characterized by

$$
\begin{aligned}
& \dot{x}_{t}^{*}(t)=\frac{K_{1}}{(\hat{T}-t)^{\frac{3}{2}}}, \\
& x^{*}(t)=\frac{K_{1}}{(\hat{T}-t)^{\frac{1}{2}}}+K_{2},
\end{aligned}
$$

where

\section{Proof: see Kalife and Mouti (2017)}

We remind that the mean cost optimal strategy for the equity case is characterized by having a constant trading rate $\dot{x}_{t}^{*}=-\frac{X}{T}$, as shown in

$$
\begin{aligned}
& K_{1}=\frac{X}{2\left(\hat{T}^{-\frac{1}{2}}-(\hat{T}-T)^{\frac{1}{2}}\right)}, \\
& K_{2}=-2 K_{1}(\hat{T}-T)^{-\frac{1}{2}} .
\end{aligned}
$$

Bertsimas and Lo (1998) in a discrete-time setting. In contrast, in the option framework under the impact function we select, the trading speed is an increasing convex function of time. 
The mean cost under the Black and Scholes framework is the only case where a closed solution can be found, is illustrated below for $\mathrm{t}=1$, $\mathrm{T}=0.5, \mathrm{X}=-1$ :

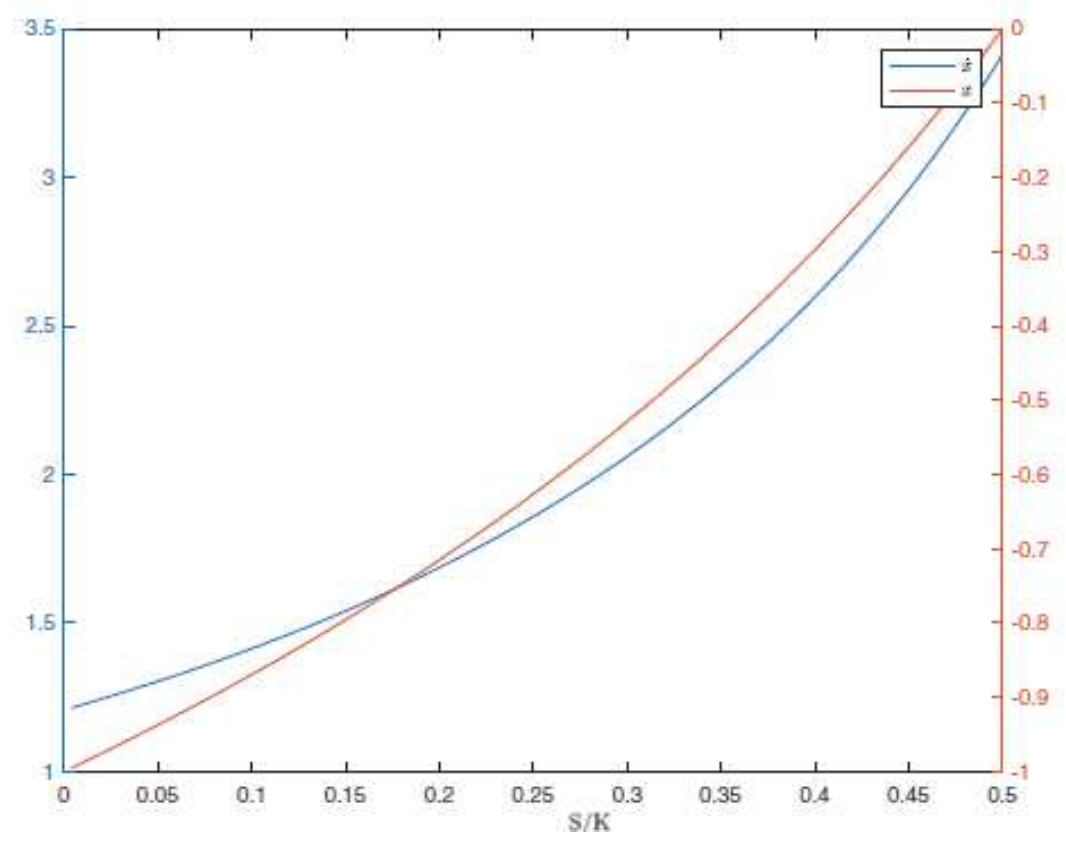

Fig. 6. The mean cost optimal strategy under the Black and Scholes model

In summary, the optimal execution strategy to maximize mean profit/loss provides a rather stable pace of trading as illustrated in the figure below, depending only mildly on the stock price path: the pace is rather constant at the beginning and then gradually increases as it gets close to maturity, which is intuitive given the fixed quantity to buy within a fixed time period, implying the insurer must acquire at a faster rate as time passes.

In the following sections, we develop the optimal execution framework under a risk/reward criterion, where the optimal strategy turns out to be more sensitive to the underlying price evolution, urging the agent to trade faster at the beginning of the strategy.

2.1.3. The optimal execution strategy significantly depends on the risk appetite: the mean-variance framework. The mean cost is usually used for an agent who does not monitor the risk of his strategy. Investors, however, usually takes into account their risk aversion, through utility functions or using risk/reward criterion such as the mean-variance. The literature on these problems is rich for optimal execution of a book of equity shares. For example Almgren and Chriss (2000) and Forsyth (2010) studied the mean-variance optimal execution problem. Gatheral and Schied (2011) took the timeaverage value-at-risk associated with the $P \& L$ of the position, while Forsyth et al. (2011) used a quadratic variance as a risk criterion. In this paper, we focus on the mean-variance criterion in light of Almgren (2012) and Almgren and Chriss (2000). The meanvariance of the cost of trading is defined by: $E[C(x)]+\lambda \operatorname{Var}[C(x)]$,

where $\lambda>0$ is the variance penalty. The choice of this coefficient cannot be explained in terms of fundamental investment preferences. The value is chosen in order to obtain solutions that bring out a certain meaning to the optimization problem.

The variance of the cost function term can be written as follows:

$$
\begin{aligned}
& \operatorname{Var}[C(x)]=E\left[\left(\int_{0}^{T} \tilde{P}_{t} \dot{x}_{t} d t-E\left[\int_{0}^{T} \tilde{P}_{t} \dot{x}_{t} d t\right]\right)^{2}\right] \\
& =E\left[\left(\int_{0}^{T} \frac{1}{2} x_{t}\left(\tilde{\sigma}_{t}^{2}-\sigma^{2}\right) S_{t}^{2} \partial_{s s} \tilde{P}\left(t, S_{t}\right) d t-\int_{0}^{T} x_{t} \sigma S_{t} \partial_{s} \tilde{P}\left(t, S_{t}\right) d W_{t}\right)^{2}\right] \\
& =E\left[\int_{0}^{T} x_{t}^{2} \sigma^{2} S_{t}^{2} \partial_{s} \tilde{P}^{2}\left(t, S_{t}\right) d t\right] \\
& =\{\text { terms arising from uncertainty in the drift part }\} .
\end{aligned}
$$


The exact expression of the variance is complicated since all terms are random. A reasonable assumption is that the largest source of uncertainty arises from the stochastic integral part. Lozenz and Almgren (2011) and Tse et al. (2013) argued that the terms in the drift part are small compared with market dynamics, which we verified numerically in our case.

In this section, we are interested in the price impact formulation with temporary impact only. That is, we can easily deduce that the mean-variance objective function can be approximated as the following:

$$
E[C(x)]+\lambda \operatorname{Var}[C(x)] \approx E\left[\int_{0}^{T} \frac{1}{2} \tilde{\eta} \sigma \dot{x}_{t}^{2} S_{t}^{2}(\hat{T}-t)^{\frac{3}{2}} \Gamma\left(t, S_{t}\right) d t+\tilde{\lambda} \int_{0}^{T} x_{t}^{2} \sigma^{2} S_{t}^{2} \Delta^{2}\left(t, S_{t}\right) d t\right] .
$$

We then set up the dynamic programming problem. To do so, we parameterize strategies $\dot{x}$ by their speed of trading and define $\alpha$ the control as $-\dot{x}_{t}$.
We introduce $\mathrm{A}(\mathrm{T} ; \mathrm{X})$ the class of all progressively measurable processes $\left(\alpha_{t}\right), \quad 0 \leq t \leq T$ for which the parameterized strategy defined by

$$
x_{t}^{\alpha}:=X-\int_{0}^{t} \alpha_{s} d s, 0 \leq t \leq T
$$

verifies necessary conditions of integrability.

We define the value function

$$
\begin{aligned}
& U(t, S, x)= \\
& \inf _{\alpha \in A(T, X)} E_{t}\left[\int_{0}^{T}\left\{\alpha_{u}^{2} S_{u}^{2}(\hat{T}-u)^{\frac{3}{2}} \Gamma\left(u, S_{u}\right)+\lambda \sigma^{2}\left(x_{u}^{\alpha}\right)^{2} S_{u}^{2} \Delta^{2}\left(u, S_{u}\right)\right\} d u\right] .
\end{aligned}
$$

We restrict our framework to Markovian controls, i.e., the agent's optimal trading speed at time $t$ is completely determined by the current state. Using the standard procedure of deriving the HJB equation in stochastic control problems (see Yong \& Zhou, 1999), the solution to the reduced optimization problem solves the PDE:

$$
\partial_{t} U+\frac{1}{2} \sigma^{2} S^{2} \partial_{s s} U+\tilde{\lambda} x^{2} S^{2} \Delta^{2}(t, S)+\inf _{a \in R}\left\{\alpha^{2} S^{2}(\hat{T}-t)^{\frac{3}{2}} \Gamma(t, S)-\alpha \partial_{x} U\right\}=0 .
$$

The so-called finite-fuel constraint required from strategies (i.e., $\int_{0}^{T} \alpha_{t} d t=X \quad$ ) suggests the value

function $U$ should satisfy a singular terminal condition of the form

$$
\lim _{t \rightarrow T} U(t, S, x)=\left\{\begin{array}{ll}
0 & \text { if } x=0 \\
+\infty & \text { if } x \neq 0
\end{array} .\right.
$$

To solve the problem numerically and justify the following the parameterized problem with a finite existence and uniqueness of the solution, we set the terminal condition

$$
\begin{gathered}
U_{\varepsilon}(t, S, x)=\inf _{x)} E_{t}\left[\int_{0}^{T}\left\{\dot{x}_{u}^{2} S_{u}^{2}(\hat{T}-u)^{\frac{3}{2}} \Gamma\left(u, S_{u}\right)+\lambda \sigma^{2}\left(x_{u}\right)^{2} S_{u}^{2} \Delta^{2}\left(u, S_{u}\right)\right\} d u+\frac{1}{\varepsilon \psi}\left(x^{\alpha} T\right)\right] \\
U_{\varepsilon}(T, S, x)=\frac{1}{\varepsilon} \psi(x) \begin{cases}0 & \text { if } x=0 \\
\gg 1 & \text { if } x \neq 0 .\end{cases}
\end{gathered}
$$

Substituting the infinite limit by the large penalty as above on the value function as $t \rightarrow T$ not only forbids to trade a large quantity at the end time, but also allows to have a regular control variable which can be solved numerically.

Then we reparameterize the controlled state variable $x_{t}^{k}$ by its rate of trading $\mathrm{k}$ such that: 
Insurance Markets and Companies, Volume 8, Issue 1, 2017

$$
d x_{t}^{k}=-k_{t} x_{t}^{k} d t
$$

Using such parameterization, we reduce the value function:

$$
U_{\varepsilon}(t, s, x)=: x^{2} u_{\varepsilon}(t, s),
$$

where

$$
\begin{gathered}
u_{\varepsilon}(t, S)=\inf _{k \in K} E_{t}\left[\int_{t}^{T} e^{-\int_{t}^{u} 2 k_{s} d s}\left\{k_{u}^{2}(\hat{T}-u)^{\frac{3}{2}} S_{u}^{2} \Gamma\left(u, S_{u}\right)+\lambda \sigma^{2} S_{u}^{2} \Delta^{2}\left(u, S_{u}\right)\right\} d u+\frac{1}{\varepsilon} e^{-\int_{t}^{T} k_{s} d s}\right] \\
\left\{\begin{array}{l}
\partial_{t} u_{\varepsilon}^{*}+\frac{1}{2} \sigma^{2} S^{2} \partial_{S S} u_{\varepsilon}^{*}+\lambda \sigma^{2} S^{2} \Delta^{2}(t, S)-\frac{1}{(\hat{T}-t)^{\frac{3}{2}} S^{2} \Gamma(t, S)} u_{\varepsilon}^{2}=0 \\
u_{\varepsilon}^{*}(T, s)=\frac{1}{\varepsilon} .
\end{array}\right.
\end{gathered}
$$

Theorem 2. The optimal trading rate is:

$$
k^{*}(t, S)=\frac{u_{\varepsilon}(t, S)}{(\hat{T}-t)^{\frac{3}{2}} S^{2} \Gamma(t, S)}
$$

Proof: Kalife and Mouti (2017)

Although this minimization problem does not admit a closed-form solution, the above quasi-linear PDE can be linearized by decomposing the quadratic term into the product of an explicit and implicit term. Thus, the general family of the two-level implicit schemes for this problem is:

$$
\frac{u_{j}^{n+1}-u_{j}^{n}}{\Delta \tau}=\theta L_{h} u_{j}^{n+1}+(1-\theta) L_{h} u_{j}^{n}+\frac{1}{\left(\hat{T}-t_{n}\right)^{\frac{3}{2}} S_{j}^{2} \Gamma_{j}^{n}} u_{j}^{n} u_{j}^{n+1}+\lambda \sigma^{2}\left(S_{j}\right)^{2}\left(\Delta_{j}^{n}\right)^{2}
$$

Then we can solve the problem numerically using finite differences methods as in Kalife and Mouti (2017) In our numerical experiment, we present results for a long position on ATM put options with the parameters as follows:

Table 3. Simulations inputs parameters

\begin{tabular}{|l|c|}
\hline \multicolumn{1}{|c|}{ Parameter } & Value \\
\hline$\sigma$ & $1 / 12$ (years) \\
\hline$T$ (the strategy horizon) & 1 (years) \\
\hline$\hat{T}$ (the option maturity) & 0 \\
\hline$\mu$ & 0 \\
\hline$r$ & 0 \\
\hline$S_{0}$ & $S_{0}$ \\
\hline$K$ & Buy \\
\hline Action & -1 \\
\hline$x_{0}$ & 0.05 \\
\hline$\eta$ & 4 trades per day \\
\hline Trading frequency & $0,1,10,100$ \\
\hline$\lambda$ & \\
\hline
\end{tabular}



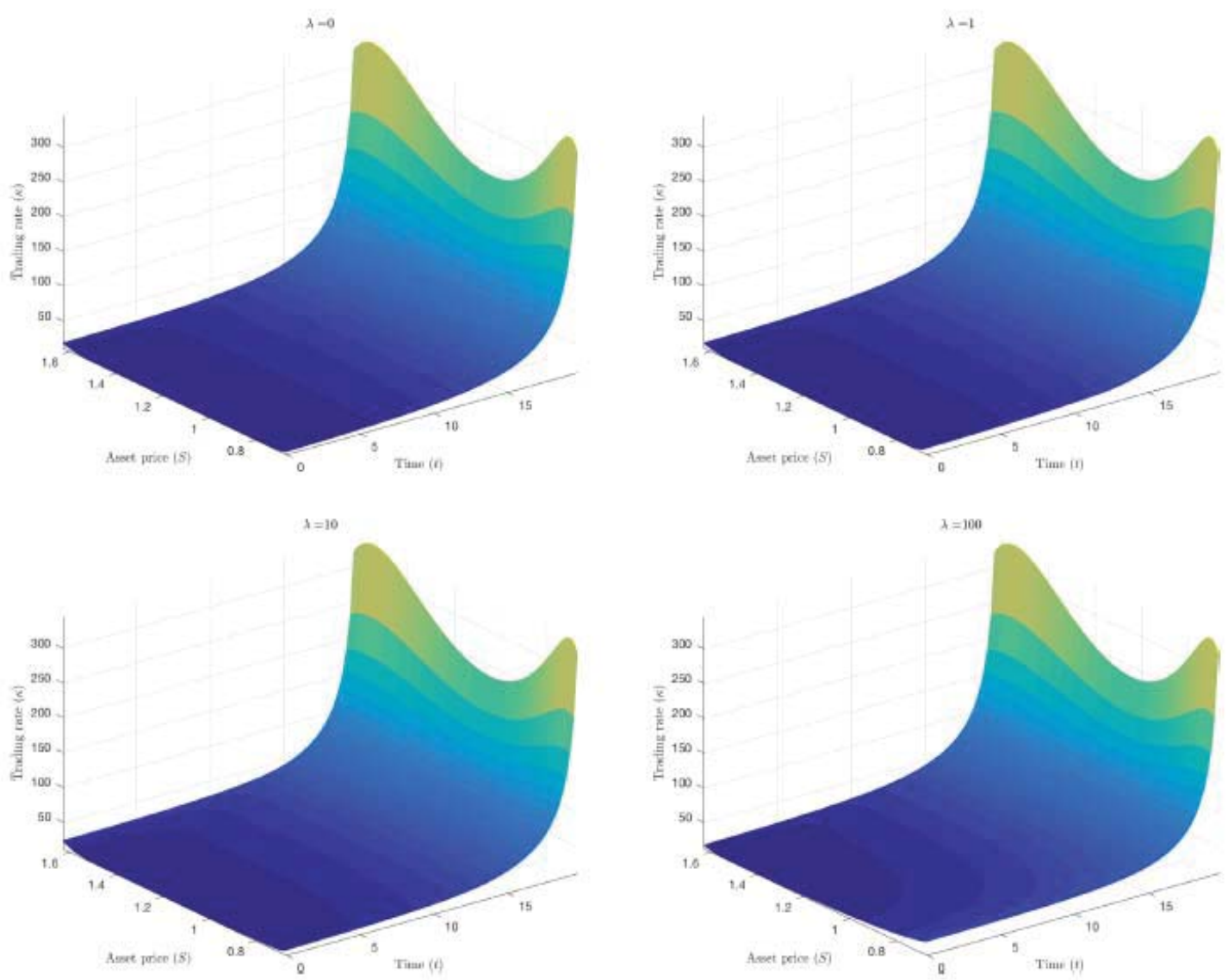

Fig. 7. The rate of trading as a function of the underlying price $S$ and time $t$ for different values of $\lambda$ : mean objective $(\lambda=0$ top left) or mean-variance ( $\lambda=1$ top right, 10 bottom left, 100 bottom right)

This chart illustrates the optimal execution strategy through the rate of trading as a function of the underlying price $\mathrm{S}$ and time $\mathrm{t}$. The strategy does not depend on the trader inventory position. However, as time increases the trading rate increases (convex in time): as the maturity approaches the agent must acquire faster as time passes. This increase has a shape of an inverse function of time. This is consistent with the equity case where the dynamic strategy is proportional to $1 /(\mathrm{T}-\mathrm{t})$. The rate increases as the asset price decreases (even though this seems slight in the graphics).

The mean case $(\lambda=0)$ is the least affected by the spot variation. In contrast, this representation allows to see that the mean-variance (i.e., $\lambda \neq 0$ ) with a highrisk aversion is most sensitive to price movements: the agent tends to liquidate her position at the beginning in order to reduce the P\&L variance that plays a non-negligible role in her choice. To gain additional insight, we plot below four paths of the underlying price together with the rate of trading, the inventory and quantity to be traded, where adding the variance pushes the agent to adapt the strategy to the underlying level: when as the risk aversion parameter increases, the traded quantity tends to be larger at the beginning. 

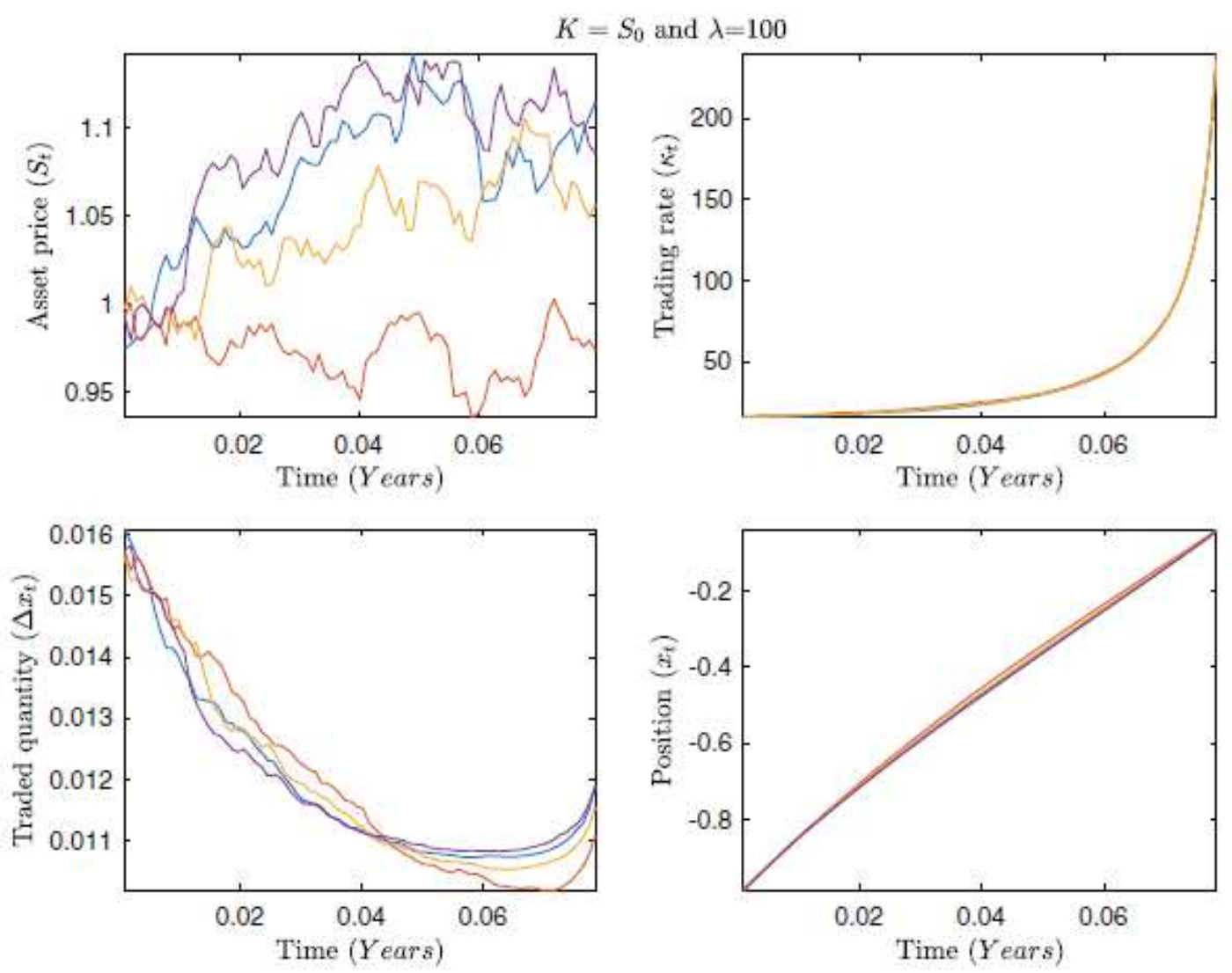

Fig. 8. Sample paths of the evolution of the fundamental price, trading rate, inventory and traded quantity throughout the execution for $\lambda=100$

In contrast, if the dispersion of the profit/loss becomes an additional driver of the risk appetite, then the optimal execution strategy significantly depends on the stock path, with a faster pace when the stock level is low compared to when the stock level is high: indeed, as the stock decreases, the cost of the put option increases.

The mean-variance profit/loss framework prevents the insurance company from waiting until maturity to trade a large quantity and instead favors a decreasing trading pace as time passes.

2.2. In case of significant delta hedging activity, the optimal execution strategy is determined by a no arbitrage framework (from the large trader's perspective) which incorporates the so-called "hedging feedback effects". We consider here the interaction of one "large trader" whose action affects prices and many price takers "small traders", the usual no arbitrage condition (Delbaen and Schachermayer (1994)) doesn't apply. We use a continuous time version of Jarrow (1994): "No Market Manipulation Strategies", which requires additional but relevant required assumptions:

- The asset price is independent of the large trader's past holdings.
- Real wealth (as if the holdings liquidated).

- Synchronous markets condition.

- Prices adjust instantaneously across underlying and derivatives.

- Absence of corners.

2.2.1. Hedging feedback effects on option price. Large dealers are net writers of options thus need to neutralize the risk by replicating synthetically options. As a result, an additional process, the number of underlying assets held by the large trader, needs to be introduced, which gives rise to nonlinear feedback effects (Frey, 1998).

Actually static hedging using options exists only for relatively short maturities. For very long dated options, dynamic replication is the only way for hedging the written options positions, by taking an offsetting position in the underlying asset. In order to manage the risk exposure, the large trader must adjust the "delta" hedge position after a price shock to allow for the change in the option's price sensitivity. As the underlying asset's price rises further, however, the option's value becomes more sensitive to changes in the underlying asset's price, which implies an increasing hedge position in the underlying asset, affecting in 
return its price process as the hedge adjustment is to buy (sell) the underlying asset after its price rises (falls).

The underlying asset price dynamics can be modeled as

$$
d \tilde{S}_{t}=\sigma_{t} \tilde{S}_{t} W_{t}+\rho_{t} \lambda\left(\tilde{S}_{t}\right) \tilde{S}_{t} d \alpha_{t}
$$

Where $\lambda$ is a continuous function called 'market liquidity profile', used to retrieve a particular shape of the implied volatility smile, while $\rho$ represents the intensity of the liquidity impact. If we now apply the Black-Scholes methodology to such modified dynamics, under a zero risk-free interest rate (for simplicity of notation), we obtain a modified BlackScholes PDE:

$$
\left\{\begin{array}{l}
u_{t}(t, \hat{S}, \gamma)+\frac{1}{2} \frac{1}{\left(1+\rho \lambda(\tilde{S}) \tilde{S} u_{\tilde{S} \tilde{S}}(t, \tilde{S}, \gamma)^{2}\right.} \sigma^{2} \tilde{S} u_{\tilde{S} \tilde{S}}(t, \tilde{S}, \gamma)=0 \\
u(T, \hat{S}, \gamma)=n h(\tilde{S})
\end{array}\right.
$$

\section{Proof: see Frey (1998)}

This modified Black-Scholes equation is a fully nonlinear parabolic PDE, requiring specific numerical implementation ensuring accuracy, flexibility and stability, as proposed by Fahim and al (2005) and adapted by Kalife, Tan and Wong (2012).

Actually, as the large trader sells European calls, she has to buy a large amount of the underlying asset in order to hedge synthetically, which makes the underlying asset price rise, thus the short delta decreases, implying a short gamma, so the 'feedback' volatility rises. Consequently the option unit price turns out to be higher than the usual pricetaker B\&S price: selling a large amount of calls causes the price to rise! But it is actually a consequence of the positive feedback effect induced by the dynamic hedging of the large trader, stemming from the absence of sufficient natural counterparts to meet the demand for puts and calls.

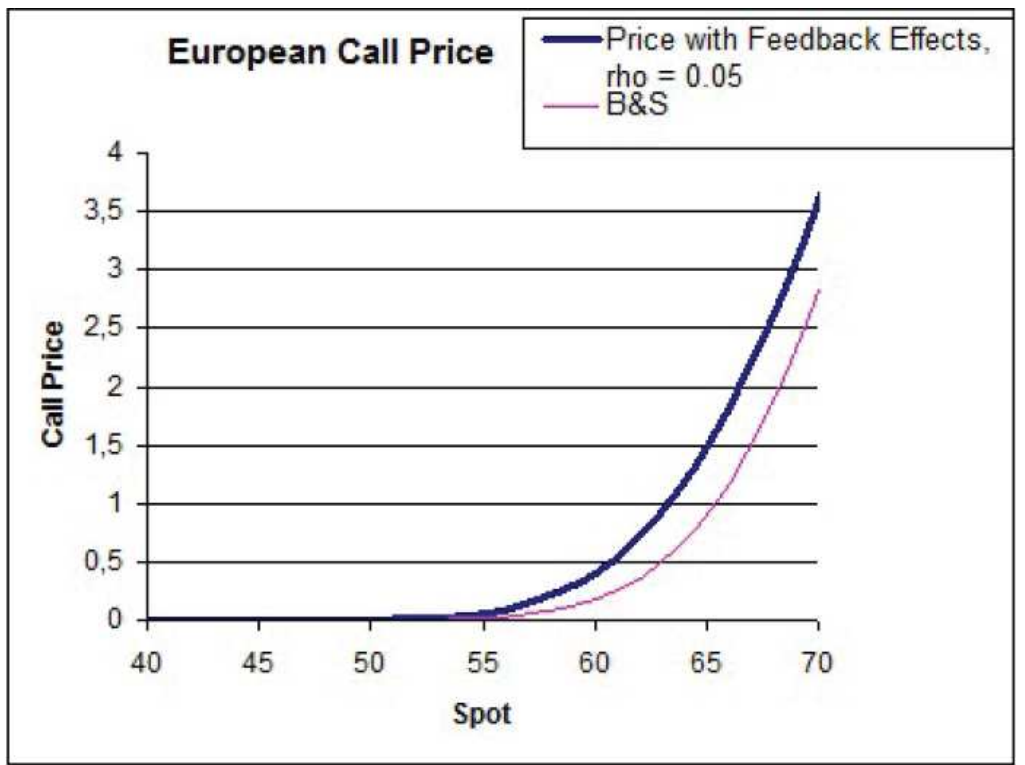

Fig. 9. European call price

2.2.2. Hedging feedback effects impact on Greeks. The gap caused by the hedging feedback effect (tracking error) is always positive, so the B\&S delta hedging strategy always implies a loss, directly linked to the difference of volatilities; growing with the gamma (i.e., the large trader hedging activity), while increasing with lower liquidity (higher $\rho$ ). 


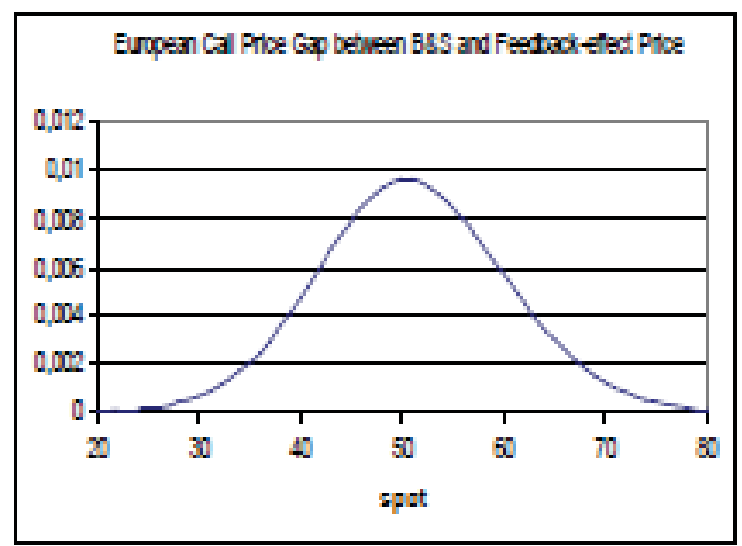

Fig. 10. European call price gap between $B \& S$ and Feedback effect price

In terms of delta hedging we distinguish three effects:

- A positive moneyness effect: the large trader buys more underlying assets for in the money calls (more likely to be exercised).

- A negative volatility effect: for in the money calls a higher volatility implies a higher

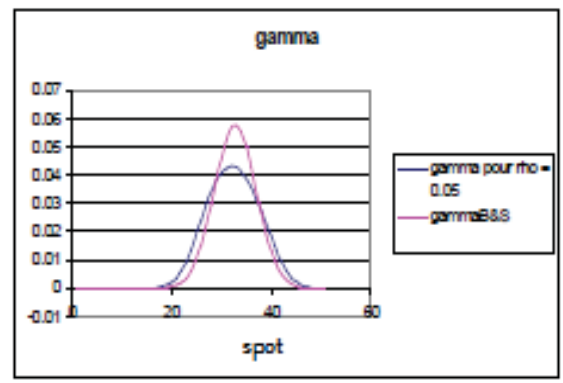

Fig. 11. The B\&S gamma hedging strategy

\section{Conclusion}

Market impact risk refers to the degree to which large size transactions can be carried out in a timely fashion with a minimal impact on prices. As a result managing investment and liquidity risks for large players requires introducing an explicit market impact function, and applying to derivatives significantly depends on whether probability to leave out of the money, which reduces the delta.

- A negative time to maturity effect: as residual time to maturity decreases, the optimal quantity to hedge is more predictable, which reduces the delta.

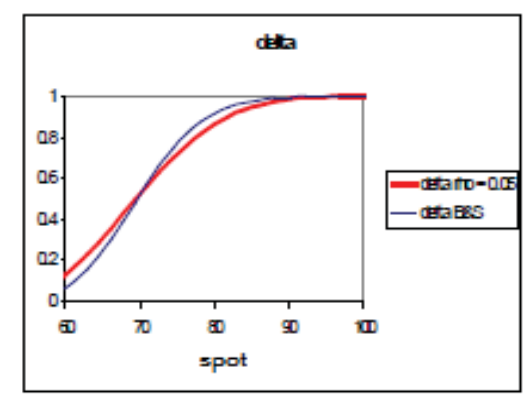

Fig. 12. The B\&S delta hedging strategy

there is or not significant delta hedging activity: in case of no significant delta hedging activity, the risk appetite has significant influence on the optimal execution strategy; while in case of significant delta hedging activity the optimal trading involves feedback hedging effects translating into a modified Black-Scholes hedging strategy.

\section{References}

1. Almgren, R. (2003). Optimal execution with nonlinear impact functions and trading enhanced risk. Applied Mathematical Finance, $10, \quad 18.18$ Retrieved $\quad$ from https://econpapers.repec.org/article/tafapmtfi/v_3a10_3ay_3a2003_3ai_3a1_3ap_3a1-18.htm

2. Almgren, R. (2012). Optimal trading with stochastic liquidity and volatility. SIAM Journal on Financial Mathematics, 3, 163-181. Retrieved from http://epubs.siam.org/doi/10.1137/090763470

3. Almgren, R., \& Chriss, N. (2000). Optimal execution of portfolio transactions. (Working paper). Retrieved from https://www.researchgate.net/publication/2327914

4. Bertsimas, D., \& Lo, A. W. (1998). Optimal control of execution costs. Journal of Financial Markets, 1, 1-50. Retrieved from https://www.sciencedirect.com/science/article/pii/S1386418197000128

5. Chan, L., \& Lakonishok, J. (1995). The behavior of stock prices around institutional trades. Journal of Finance, 50, 1147-1174. Retrieved from http://onlinelibrary.wiley.com/doi/10.1111/j.1540-6261.1995.tb04053.x/abstract

6. Christoffersen, P., Goyenko, R., Jacobs, K. \& Karoui, M. (2014). Illiquidity premia in the equity options market. Retrieved from https://papers.ssrn.com/sol3/papers.cfm?abstract_id=1784868 
7. Forsyth, P. A. (2010). A Hamilton Jacobi Bellman approach to optimal trade execution (Working paper). Retrieved from https://cs.uwaterloo.ca/ paforsyt/optimal_trade.pdf

8. Forsyth, P. A., Kennedy, J. S., Tse, S. T., \& Windcliff, H. (2011). Optimal trade execution: A mean-quadraticvariation approach. Journal of Economic Dynamics and Control, 36(12), 1971-1991. Retrieved from https://www.sciencedirect.com/science/article/pii/S0165188912001236

9. Frey, R. (1998). Perfect option hedging for a large trader. Finance and Stochastics, 2, 115-141. Retrieved from https:/link.springer.com/article/10.1007/s007800050035?no-access=true

10. Gârleanu, N., Pedersen, L. H., \& Poteshman, A. M. (2009). Demand-based option pricing. The Review of Financial Studies, 22(10), 4259-4299. Retrieved from https://papers.ssrn.com/sol3/papers.cfm?abstract id=676501

11. Gatheral, J., \& Schied, A. (2011). Optimal trade execution under geometric Brownian motion in the Almgren and Chriss framework. The International Journal of Theoretical and Applied Finance, 14(3), 353-368. Retrieved from https://papers.ssrn.com/sol3/papers.cfm?abstract_id=1654151

12. Huberman, G., \& Stanzl, W. (2004). Price manipulation and quasi-arbitrage. Econometrica, 72(4), 1247-1275. Retrieved from http://onlinelibrary.wiley.com/doi/10.1111/j.1468-0262.2004.00531.x/abstract

13. Jaimungal, S., \& Kinzebulatov, D. (2014). Optimal execution with a price limiter, Risk. Retrieved from https://papers.ssrn.com/sol3/papers.cfm?abstract id=2199889

14. Kalife, A., Tan, X. \& Wong, L. (2012). Dynamic hedging by a large player: from theory to practical implementation. Neural, Parallel, and Scientific Computations, 20, 191-214.

15. Kalife, A., Mouti S., Tan, X. (2015). Minimizing market impact of hedging insurance liabilities within risk appetite constraints. Insurance Markets and Companies: Analyses and Actuarial Computations, 6(2). Retrieved from https://businessperspectives.org/journals/insurance-markets-and-companies/issue-206/minimizing-marketimpact-of-hedging-insurance-liabilities-within-risk-appetite-constraints

16. Kalife, A., Mouti, S., (2017). On Optimal Options Book Execution Strategies with Market Impact. $\begin{array}{lllll}\text { Market } \quad \text { Microstructure } & \text { and } & \text { Riquidity, } & \text { Retrieved }\end{array}$ http://www.worldscientific.com/doi/abs/10.1142/S2382626617500022

17. Keim, D. B., \& Madhavan, A. (1995). Execution costs and investment performance: An empirical analysis of institutional equity trades (Working paper). School of Business Administration, University of Southern California. Retrieved from https://papers.ssrn.com/sol3/papers.cfm?abstract id=5846

18. Leland, H. E. (1985). Option pricing and replication with transaction costs. The Journal of Finance, 40(5), 12831301. Retrieved

from https://www.researchgate.net/publication/4767581_Option_Pricing_and_Replication_With_Transaction_Costs

19. Lepinette, E., \& Quoc, T. T. (2014). Approximate hedging in a local volatility model proportional transaction costs. Applied Mathematical Finance, 21(4). Retrieved from https://papers.ssrn.com/sol3/papers.cfm?abstract_id=2241023

20. Lozenz, J., \& Almgren, R. (2011). Mean-variance optimal adaptive execution. Applied Mathematical Finance, 18, 311-323. Retrieved from https://econpapers.repec.org/article/tafapmtfi/v_3a18_3ay_3a2011_3ai_3a5_3ap_3a395422.htm

21. Tse, S. T., Forsyth, P. A., Kennedy, J. S., \& Windcliff, H. (2013). Comparison between the mean-variance optimal and the mean-quadratic-variation optimal trading strategies. Applied Mathematical Finance, 20(5), 415-449. Retrieved from https://econpapers.repec.org/article/tafapmtfi/v_3a20_3ay_3a2013_3ai_3a5_3ap_3a415-449.htm

22. Yong, J., \& Zhou, X. Y. (1999). Stochastic Controls: Hamiltonian Systems and HJB Equations. Springer. 